The Astrophysical Journal, 245:512-528, 1981 April 15

(C) 1981. The American Astronomical Society. All rights reserved. Printed in U.S.A.

\title{
TEMPERATURES OF GALACTIC MOLECULAR CLOUDS SHOWING CO SELF-ABSORPTION
}

\author{
T. G. Phillips AND G. R. KNAPP \\ Owens Valley Radio Observatory, California Institute of Technology \\ P. J. HugGins \\ Department of Physics, New York University \\ M. W. WERNER \\ NASA Ames Research Center, Moffett Field \\ P. G. WANNIER \\ Owens Valley Radio Observatory, California Institute of Technology \\ AND \\ G. Neugebauer And D. EnNis \\ Palomar Observatory, California Institute of Technology \\ Received 1980 August 20; accepted 1980 October 22
}

\begin{abstract}
The CO $J=2-1$ line has been observed and, in most cases, mapped in 10 star-forming molecular clouds (W3, NGC 1333, NGC 2071, Mon R2, CRL 961, $\rho$ Oph, W49N, W51A, DR 21, and Cep A). The CO $J=3-2$ line has been observed in W3 and DR 21. The CO lines from all of these sources are strongly self-absorbed. By comparing our results with published $\mathrm{CO}(1-0)$ line profiles, we find that large corrections to the temperatures of the cloud cores, as measured by the CO (1-0) lines, are required. The corrections for self-absorption bring the $\mathrm{CO}$ brightness temperatures into closer agreement with the grain temperatures inferred from far-infrared photometry.

The absorbing gas appears to have a variety of geometrical relationships with the absorbed 'background' gas for this group of sources; in one or two cases there may be no physical connection between the absorbed and absorbing regions. For some $\mathrm{CO}$ sources, the combined $\mathrm{CO}$ data indicate the presence of velocity gradients; however, whether these are due to expansion or collapse cannot be decided on the basis of the presently available data.
\end{abstract}

Subject headings: interstellar: molecules - nebulae: general

\section{INTRODUCTION}

It is well known that the dust and gas temperatures of molecular clouds, as measured by infrared continuum and $\mathrm{CO}$ line observations, respectively, do not always agree. In the case of the Orion molecular cloud, both components give an average temperature of $\sim 75 \mathrm{~K}$ in the $1^{\prime}$ region around the $\mathrm{BN} / \mathrm{KL}$ cluster (Liszt et al. 1974; Werner et al. 1976). However, for many other sources this is not the case. For W3, for example, the dust temperature is $65 \mathrm{~K}$ (Werner et al. 1981), while the CO (1-0) brightness temperature is $\sim 40 \mathrm{~K}$ (Snell and Loren 1977). In this paper, which describes an observational study of molecular clouds showing CO selfabsorption, we show that the absorption effects are more important than have previously been assumed. Corrections for self-absorption effects bring the gas temperatures into approximate agreement with the dust temperatures.

There are several reasons for believing that the millimeter-wavelength rotational lines of the $\mathrm{CO}$ molecule are highly saturated for most galactic molecular clouds.
The ${ }^{12} \mathrm{CO}$ and ${ }^{13} \mathrm{CO}$ lines have antenna temperature ratios much lower than the terrestrial ${ }^{12} \mathrm{C} /{ }^{13} \mathrm{C}$ isotope ratio value. Second, the line antenna temperatures do not increase as the upper $J$-value of the transition increases, as would be the case for low-opacity clouds. Third, the line widths increase with increasing opacity (caused either by an increase in $J$ or by increasing isotope abundance), as for a curve of growth. Using this, Phillips et al. (1979) have found optical depths of $\sim 100$ for the ${ }^{12} \mathrm{C}^{16} \mathrm{O}(J=1-0)$ line from several clouds.

For these strongly saturated lines, one might expect the line profile to have a reversal dip in many cases, since the molecule excitation temperature may have a line-of-sight gradient. This may occur even in clouds with uniform kinetic temperature, due to the different intensities of the radiation field in the center and on the outside of the cloud (e.g., Leung and Liszt 1976; Kwan 1978). The lack of observed self-absorption in many CO clouds was one of the factors which led to the adoption of the large velocity gradient (LVG) model for molecular clouds (Scoville and Solomon 1974; Goldreich and 
Kwan 1974) in which a systematic line-of-sight velocity gradient through the cloud results in no two parts of the cloud along a line of sight being at the same radial velocity.

A second situation which could give rise to selfabsorption is that in which a 'cold' cloud (i.e., a cloud of relatively low excitation temperature) lies somewhere along the line of sight to a hotter cloud with the same radial velocity. Since clouds in which a large region has a $\mathrm{CO}$ brightness temperature much above $\sim 10 \mathrm{~K}$ are those in the immediate vicinity of a heating source, such as embedded stars or an $\mathrm{H}$ II region, self-absorbed $\mathrm{CO}$ lines might well be expected for such regions, with the cold absorbing cloud being in the neighborhood of the hot cloud or elsewhere along the line of sight to the cloud. Indeed, all known cases of $\mathrm{CO}$ self-absorption are toward strongly heated sources (Leung and Brown 1977).

The present paper reports sensitive, high spectral resolution observations of the $\operatorname{CO} J=2-1$ line toward several galactic star-forming molecular clouds. Also, in two cases, observations in the $\mathrm{CO} J=3-2$ lines have been made. In thermal equilibrium at moderate temperatures $(T \gtrsim 15 \mathrm{~K})$ these lines tend to have intrinsically higher opacities than does the CO $J=1-0$ line, and so might be expected to show self-absorption effects more readily. We have therefore examined the physical properties of the absorbed and absorbing gas by comparing observations of the $\mathrm{CO}(1-0),(2-1)$, and (3-2) lines. The relative geometry of the absorbed and absorbing gas has also been studied by mapping the centers of several of the molecular clouds in the CO $J=2-1$ line.

\section{OBSERVATIONS}

Most of the observations discussed in this paper were made of the $\mathrm{CO}(2-1)$ line at $230 \mathrm{GHz}$ using a hotelectron bolometer receiver at the Cassegrain focus of the Caltech 10 meter telescope (Leighton 1978). The data taking and calibration procedures are described by Phillips et al. (1979). It was assumed that all of the clouds observed are much larger than the $26^{\prime \prime}$ beam of the telescope, so that the extended source beam efficiency of 0.65 found from observations of the Moon was used in calculating $T_{A}^{*}$, the Rayleigh-Jeans equivalent brightness temperature of the $\mathrm{CO}(2-1)$ lines.

The observations were made in the total power mode, switching between the source and a reference position on the sky. All of the reference points were first observed to make sure that they were free of $\mathrm{CO}$ emission, and no emission was found to a sensitivity of $\sim 2 \mathrm{~K}$ from any reference point. The total integration time for an individual spectrum was of order 20 minutes. The receiver system temperature was $200-300 \mathrm{~K}$. Some of the observations were made in 1978 April-May, and the majority in 1978 November.

Several points were observed in most clouds. To minimize point-to-point calibration variations due to drifts in the receiver gain and atmospheric opacity, each map point, plus the 'off' position, was observed in sequence, the sequence being repeated several times. The atmospheric opacity during most of the observations made in 1978 November was high, with values of the zenith opacity between 0.6 and 1.0. Partly for this reason, and also because of possible small-scale temperature structure in the clouds, our temperature scale is only accurate to $\sim 30 \% \mathrm{rms}$, although our observations agree well with $\mathrm{CO}(2-1)$ observations of some of the same objects by Israel et al. (1981) which were made using the same telescope but a different receiver. Altogether, 10 dark clouds or $\mathrm{H}$ in regions known or suspected of having a self-absorption feature were observed. Most of these were mapped in right ascension or declination, or both, at angular spacings of $30^{\prime \prime}$ or $60^{\prime \prime}$. The pointing accuracy was $\lesssim 10^{\prime \prime}$.

Observations of the $\mathrm{CO}(3-2)$ line at $345.8 \mathrm{GHz}$ were made of W3 and of DR 21 in 1977 February using a hot-electron bolometer receiver at the prime focus of the Hale $5 \mathrm{~m}$ telescope. The receiver bandwidth used was 1 $\mathrm{MHz}\left(=0.9 \mathrm{~km} \mathrm{~s}^{-1}\right.$ at $\left.0.87 \mathrm{~mm}\right)$. The telescope beamwidth at this wavelength was $36^{\prime \prime}$. The observational techniques are described in detail by Phillips et al.

TABLE 1

Central Positions of Clouds Observed in the CO (2-1) Line

\begin{tabular}{|c|c|c|c|c|}
\hline Source & $\alpha(1950)$ & $\delta(1950)$ & $\begin{array}{l}\text { Bandwidth } \\
\left(\mathrm{km} \mathrm{s}^{-1}\right)\end{array}$ & Reference for CO (1-0) Data \\
\hline $\mathrm{W}^{\mathrm{a}}$. & $02^{\mathrm{h}} 21^{\mathrm{m}} 55^{\mathrm{s}} .0$ & $+61^{\circ} 51^{\prime} 51^{\prime \prime}$ & 1.3 & Snell and Loren 1977 \\
\hline NGC 1333 & $\begin{array}{lll}03 & 25 & 56.0\end{array}$ & $+31 \quad 1038$ & 0.7 & Loren 1976 \\
\hline NGC $2071 \ldots$ & $\begin{array}{lll}05 & 44 \quad 30.3\end{array}$ & +002018 & 0.7 & Loren and Wootten 1980 \\
\hline Mon R2 ...... & $\begin{array}{lll}06 & 05 & 20.0\end{array}$ & -062230 & 0.7 & Snell and Loren 1977 \\
\hline CRL 961 & $\begin{array}{lll}06 & 31 & 59.0\end{array}$ & +041509 & 0.7 & Blitz 1980 \\
\hline$\rho$ Oph . & $\begin{array}{lll}16 & 23 & 35.0\end{array}$ & $-24 \quad 1900$ & 0.7 & \\
\hline W49N. & $\begin{array}{lll}19 & 07 & 54.0\end{array}$ & +090101 & 1.3 & Mufson and Liszt 1977 \\
\hline W51A. & $\begin{array}{lll}19 & 21 & 27.0\end{array}$ & +142430 & 0.7 & Mufson and Liszt 1979 \\
\hline DR $21^{a}$ & $\begin{array}{lll}20 & 37 & 13.0\end{array}$ & +420851 & 0.7 & Dickel et al. 1978 \\
\hline Cep A & $2254 \quad 26.5$ & +614437 & 0.7 & Sargent 1977 \\
\hline
\end{tabular}

${ }^{\mathrm{a}} \mathrm{CO}(3-2)$ emission also observed. 
(1977). These observations will be compared with the CO (2-1) observations of the same sources in $\S \mathrm{IV}$, where we also compare our observations with data on the CO (1-0) line taken from the literature. All of the CO (1-0) observations cited except that of NGC 1333 were made with the NRAO 11 meter telescope, whose beamwidth at $115 \mathrm{GHz}$ is $65^{\prime \prime}$.

In Table 1, we list the sources observed. For each object, the 1950 coordinates of the central position, the velocity resolution used for the $\mathrm{CO}(2-1)$ observations, and the literature reference for the $\mathrm{CO}(1-0)$ observations are given.

\section{MODELS}

We propose to discuss the observations of each of the sources by comparing them with the predictions of simple line formation models, to be described in this section.

The occurrence of reversals due to self-absorption in the $\mathrm{CO}$ lines is by no means a universal phenomenon, and as Leung and Brown (1977) have pointed out, all of the observed cases of $\mathrm{CO}$ self-absorption in the (1-0) line occur in directions toward embedded stars in molecular clouds. This suggests that to produce observable reversals in the $\mathrm{CO}$ lines under the physical conditions obtaining in molecular clouds, a kinetic temperature gradient is required, with physically colder gas in front of hotter gas. For this reason, we have interpreted our observations in terms of a model consisting of a constant temperature hot background cloud with a colder foreground cloud at the same radial velocity, and also of constant temperature. This model is an approximation to the situation where absorption is produced by a cold cloud distant from, and not physically associated with, a hot cloud, as well as the situation in which a single cloud has its kinetic temperature decreasing outward.

In these models, we calculate only the central depth of the reversal dip and do not consider the line shapes or the line widths. The depths of the reversal dips are calculated for each of the observed lines and comparison with the observations are used to derive the temperatures for the background and foreground clouds. We have also used these calculations to decide whether the observations are in fact true cases of reversed lines or whether the appearance of such a line is mimicked by two emission components at slightly different central velocities.

We have also considered the appearance of a selfreversed line profile as a function of relative position in a cloud in two simple geometric cases: (1) with the absorbing gas forming the outer layer of a hotter cloud, and (2) with the absorbing and emitting clouds being separate.

Although much work has been presented in the literature which uses sophisticated radiative transfer models to calculate the line profiles expected in such cases in detail, we have chosen to use simple hypothetical situations for comparison with our data because we are interested in discovering the gross, and in most cases, qualitative, properties of the observed clouds. As will become obvious from our observations, the available data for many of these molecular clouds are not yet sufficiently detailed to constrain more complicated models.

\section{a) LTE Model}

Consider a $\mathrm{CO}$ cloud of uniform temperature $T_{k}=$ $T_{01}=T_{12}=T_{23}$, etc., in front of a hot background line source of temperature $T_{\mathrm{BG}}$ at the central velocity of the colder cloud. The line temperature $T_{A}^{*}$ observed inside the absorption dip produced by the cold cloud is

$$
T_{A}^{*}=\mathscr{T}\left(T_{B}\right)=\mathscr{T}\left(T_{\mathrm{BG}}\right) e^{-\tau_{c}}+\mathcal{T}\left(T_{k}\right)\left(1-e^{-\tau_{c}}\right),
$$

where $\tau_{c}$ is the optical depth of the cold cloud at the velocity of observation, and

$$
\mathscr{T}(T)=\frac{h \nu}{k}\left[\left(\exp \frac{h \nu}{k T}-1\right)^{-1}-\left(\exp \frac{h \nu}{k T_{\mathrm{BB}}}-1\right)^{-1}\right],
$$

where $T_{\mathrm{BB}}=2.7 \mathrm{~K}$ is the temperature of the universal microwave background radiation. The effect of this background for the observations described herein is very small.

The peak optical depth for a cloud of column density $N_{\text {CO }}$ is:

$$
\tau_{J+1, J}=\frac{4 \pi^{3} \mu^{2} \nu}{3 k T_{k}}\left(1-e^{-h \nu / k T_{k}}\right) e^{-h B J(J+1) / k T_{k}} \frac{N_{\mathrm{CO}}}{\Delta V},
$$

where a triangular line shape of width $\Delta V$ is assumed, and where the velocity dispersion of the foreground cloud is small compared to that of the background cloud. Away from the absorption dip, $T_{A}^{*} \approx \mathcal{T}\left(T_{\mathrm{BG}}\right)$.

In Figure 1, we show an example of the variation of the line temperature at the depth of the self-absorption dip as a function of $N_{\mathrm{CO}} / \Delta V$, where $N_{\mathrm{CO}}$ is the total CO column density in $\mathrm{cm}^{-2}$ and $\Delta V$ is the width of the $\mathrm{CO}$ line in $\mathrm{km} \mathrm{s}^{-1}$. For the model of Figure 1, the brightness temperature of the background emission was taken as 64 $\mathrm{K}$ and the kinetic temperature of the foreground cloud as $15 \mathrm{~K}$. It can be seen from equations (1)-(3) and Figure 1 that, for temperatures $\gtrsim 15 \mathrm{~K}$, the higher frequency $\mathrm{CO}$ lines will always have deeper dips than the lower frequency lines, until the optical depths in the cold cloud are so high that the line temperatures in all transitions is $\mathscr{T}\left(T_{K}\right)$. Even for saturation, the RayleighJeans equivalent brightness temperature $T_{A}^{*}$ (the measured quantity) decreases with increasing frequency (eq. 


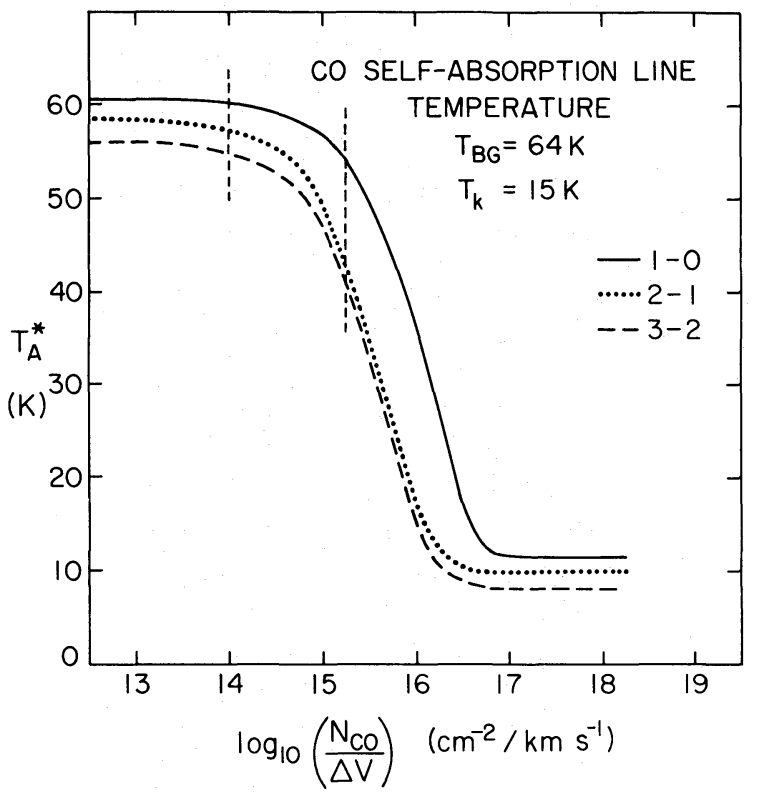

FIG. 1.- The temperature of the CO line in the center of the self-absorption dip produced by a cold cloud in LTE at temperature $15 \mathrm{~K}$ in front of a hotter cloud of temperature $64 \mathrm{~K}$, as a function of the CO column density $N_{\mathrm{CO}}$ in $\mathrm{cm}^{-2}$ per unit line width $\Delta V$ in $\mathrm{km} \mathrm{s}^{-1}$. Over the range of column densities indicated, strong reversals are seen in the (2-1) and (3-2) lines, but not in the $(1-0)$ line.
[2] and Fig. 1). The models also show that there is a large range of cold cloud column densities $\left(10^{14} \lesssim\right.$ $N_{\text {Co }} / \Delta V \lesssim 2 \times 10^{15} \mathrm{~cm}^{-2}\left[\mathrm{~km} \mathrm{~s}^{-1}\right]^{-1}$, cf. Fig. 1) over which the lower frequency lines will not show selfabsorption, while the higher frequency lines will. For $\Delta V=5 \mathrm{~km} \mathrm{~s}^{-1}$, the upper value of this range of $\mathrm{CO}$ column densities corresponds to $A_{V} \approx 0.2 \mathrm{mag}$, or to typical diffuse cloud column densities.

\section{b) Non-LTE Models}

To calculate line intensities under non-LTE conditions, the large velocity gradient (LVG) model as formulated by Goldreich and Kwan (1974) was used. For a cold, spherical model cloud of uniform density and kinetic temperature, the $\mathrm{CO}$ level populations (for seven levels, assuming the approximate collision cross sections given by Goldreich and Kwan and assuming $d V / d r=1$ $\mathrm{km} \mathrm{s}^{-1}\left(\mathrm{pc}^{-1}\right)$ are calculated; the cloud is then placed in front of a hot background line source. Examples for $T_{k}=30 \mathrm{~K}$ and $T_{k}=20 \mathrm{~K}$ ( $T_{\mathrm{BG}}=64 \mathrm{~K}$ in both cases) are shown in Figure 2. At large values of $n\left(\mathrm{H}_{2}\right)$ the line temperatures approach their LTE values. Figure 2 shows that the temperature in the dip reaches a minimum for each transition at different $\mathrm{CO}$ column densities and/or $\mathrm{H}_{2}$ densities. Since the model cloud has a velocity gradient, the dips in the various $\mathrm{CO}$ transitions will be formed at different velocities.

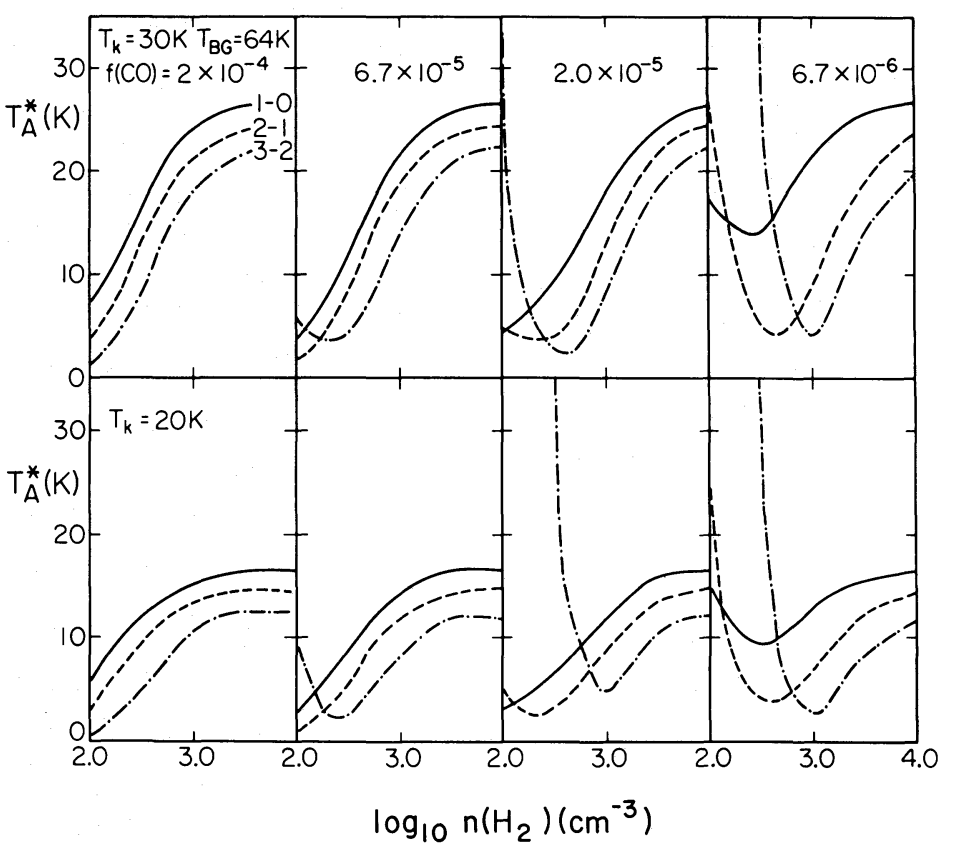

FIG. 2.-As for Fig. 1, with the cold gas in non-LTE and the LVG model used to calculate the CO level populations. The line depth is shown as a function of $n\left(\mathrm{H}_{2}\right)$ and $f(\mathrm{CO})$, the $\mathrm{CO}$ fractional abundance, for two cold cloud kinetic temperatures, $20 \mathrm{~K}$ and $30 \mathrm{~K}$. The background line temperature is again $64 \mathrm{~K}$. 


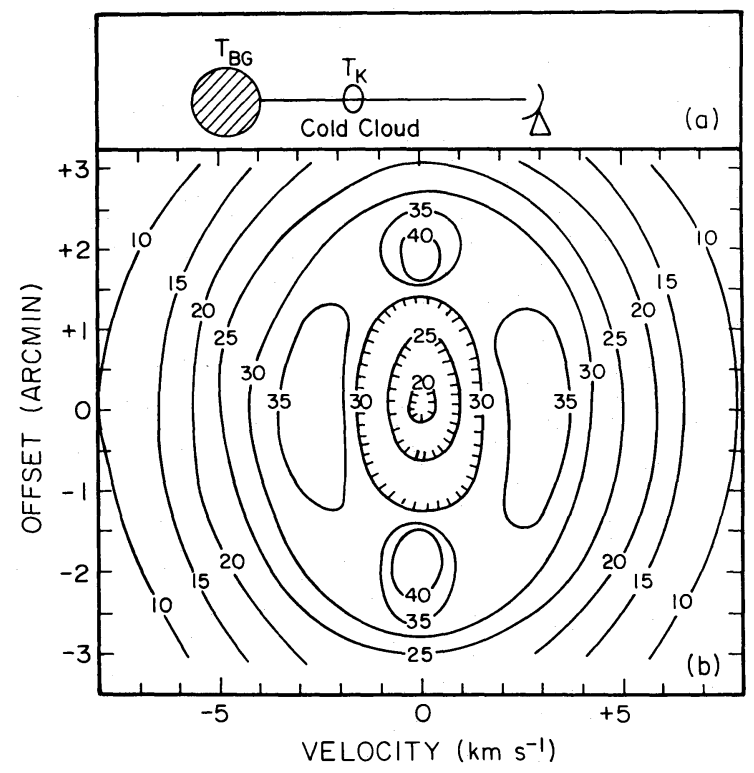

FIG. 3.-Model CO line position-velocity map (b) for the illustrated source geometry $(a)$.

\section{c) Source Geometry}

Since many of the clouds discussed in this paper have been mapped, it is in principle possible to use the maps for a qualitative examination of the source geometry. Here we show two examples: (i) a small spherical cold cloud in front of a larger background hot cloud, and (ii) absorption produced by a colder shell surrounding a hot cloud. The qualitative appearance of a CO line map in each case has been calculated assuming that the absorbing gas is in LTE (i.e., using eq. [3]) and has moderate central optical depth. For the background cloud, it has been assumed that the heating is caused by a small central embedded source, so that the brightness temperature in the $\mathrm{CO}$ line decreases away from the center. Case (i) is illustrated in Figure 3. Since the line of sight through the absorbing cloud is largest through its center, the absorption dip is deeper at the center. If the absorbing cloud were very optically thick, the temperature inside the self-absorption dip would be constant with position. Case (ii) is illustrated in Figure 4. Here the self-absorption dip is saddle-shaped, since the optical depth through the center of the cloud is a minimum. Again, if the cold gas is opaque, the temperature in the dip is constant; this time it is at the same temperature as emission seen from the edge of the cloud.

\section{d) Emission from Two Clouds}

The question arises for individual sources as to whether an apparent absorption dip is actually the minimum between two partially overlapping profiles from separate clouds at different velocities. If this were

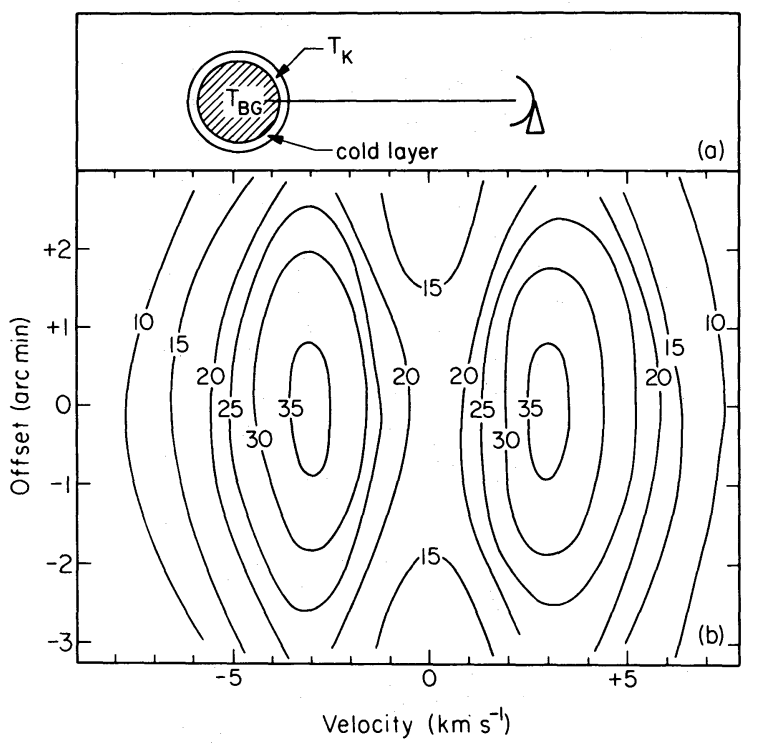

FIG. 4.-Model CO line position-velocity map $(b)$ for the illustrated source geometry $(a)$.

the case, the dip would tend to fill in as the intrinsic line opacity increases. It will be seen in our data, to be described below, that the opposite generally occurs, i.e., the dips become deeper with increasing $\mathrm{CO}$ line opacity. Also, absorption tends to create steep-sided dips; to produce such an effect with two emission profiles would require two superposed asymmetric components. Finally, the peaks of lines of much lower opacity (e.g., from the ${ }^{13} \mathrm{CO}$ or $\mathrm{C}^{18} \mathrm{O}$ molecules) occur near the velocity of the dip for absorption, while two peaks at the velocities of the ${ }^{12} \mathrm{CO}$ peaks would occur for emission from two separate clouds.

It is important to note that it is not necessarily the case that when the ${ }^{13} \mathrm{CO}$ line has a dip at the same velocity as does the ${ }^{12} \mathrm{CO}$ line that clouds at two different velocities are present. It such cases it is quite possible that the ${ }^{13} \mathrm{CO}$ line is also of high opacity and also self-absorbed. Observations of higher $J \mathrm{CO}$ lines are needed to settle the question.

\section{INDIVIDUAL SOURCES}

The observations of individual sources in this section are presented in two forms: as velocity-position maps in the CO (2-1) and (3-2) lines, and as line profiles observed in two or three $\mathrm{CO}$ transitions at the central position in each cloud. For the maps, the line intensity is plotted as a function of velocity and offset position (in right ascension or declination) from the central position given in Table 1. In these maps, the position scale has an entry for every position at which a profile was observed. The half-power diameter of the telescope beam is also shown for each map. 
We have also compared our CO (2-1) and (3-2) observations with (1-0) data taken from the literature. The main-beam efficiency of the NRAO telescope, which was used for all of these observations, is $65 \%$. We multiply the published antenna temperatures by a factor of 1.5 (cf. Ulich and Haas 1976), unless it is explicitly stated in the paper from which a $\mathrm{CO}(1-0)$ line profile is taken that this correction factor has been applied to the data. For some clouds, the maps of the CO (2-1) emission show structure on the scale of the $26^{\prime \prime}$ beam used for these observations. In these cases, the CO (2-1) profile expected for a $65^{\prime \prime}$ beam is synthesized from our data for more direct comparison with the $(1-0)$ observations.

\section{a) $D R 21$}

The CO (1-0), (2-1), and (3-2) line profiles for this source are shown in Figure 5, and maps of the $\mathrm{CO}(3-2)$ emission in Figures 6 and 7. The (3-2) profile is strongly reversed, while the $(2-1)$ profile is flattened, and the (1-0) line is peaked. The appearance of the maps suggests that the absorption seen in the CO (3-2) line is due to the colder outer edge of the cloud, although the signal-to-noise ratio for the observations is low.

If it is assumed that the $\mathrm{CO}(1-0)$ line is unabsorbed so that its brightness temperature is taken to be that of the background, no fit to the line data for the three transitions can be obtained using either LTE or nonLTE models. To fit all three line temperatures at $v=-3$ $\mathrm{km} \mathrm{s}^{-1}$ requires $T_{\mathrm{BG}} \approx 45 \mathrm{~K}$ (cf. Fig. 2) so that, despite its appearance, the (1-0) line is also strongly absorbed.

DR 21 thus provides a very striking example of the presence of significant absorption even when obvious line reversal is not present. It is likely that the CO brightness temperatures of many other sources have been severely underestimated for similar reasons.

\section{b) NGC 2071 and Mon R2}

The CO (2-1) and (1-0) line profiles for NGC 2071 are shown in Figure 8, and the velocity-declination map of the (2-1) line in Figure 9. The (2-1) and (1-0) line profiles for Mon R2 are shown in Figure 10, and the (2-1) maps in Figures 11 and 12. Both of these sources are similar in that the absorption seems to be due to diffuse foreground gas. This can be seen from the fact that the absorption dips are deepest near the cloud center, and the declination maps for both sources show that the absorption region has a limited extent. Because of the similarity of the absorption and emission velocities in both cases, the cold gas is probably part of the local complex of interstellar matter.

NGC 2071 is a small reflection nebula near M78 which is illuminated by several early B-type stars (Brown et al. 1975) and contains an $\mathrm{H}_{2} \mathrm{O}$ maser with highvelocity components (Schwartz and Buhl 1973), located

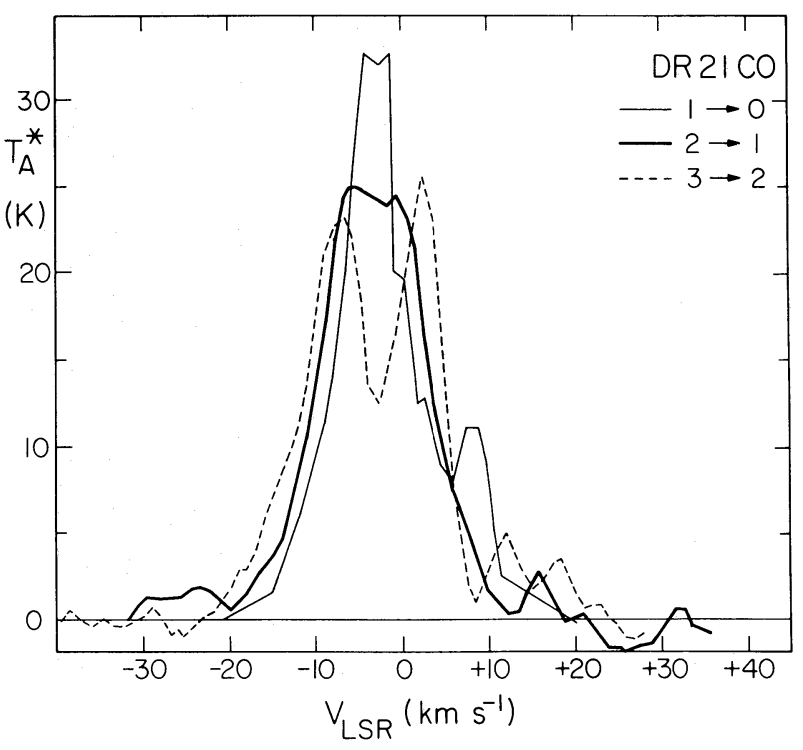

FIG. 5.-CO (1-0), (2-1), and (3-2) line profiles observed toward DR 21.

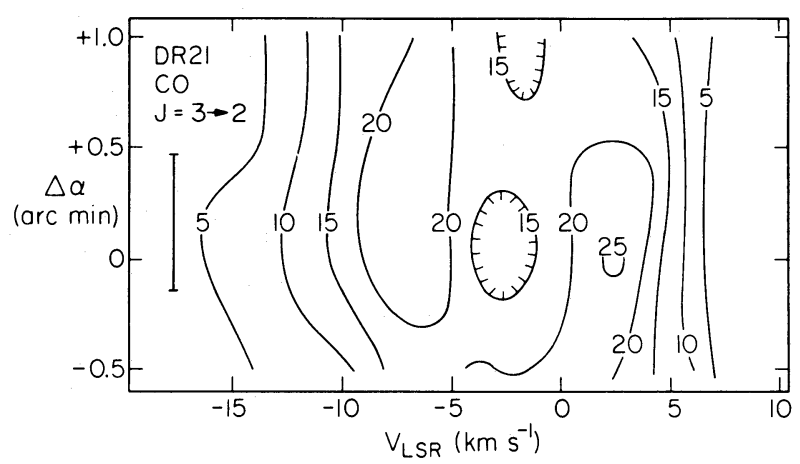

FIG. 6.- Velocity-right ascension map in the CO (3-2) line for DR 21. The half-power beam diameter is shown.

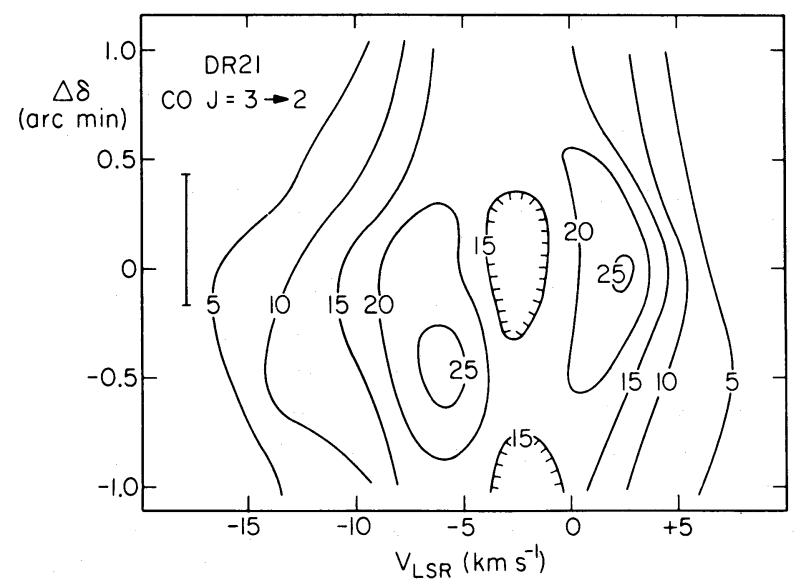

FIG. 7.-Velocity-declination map in the CO (3-2) line for DR 21. 


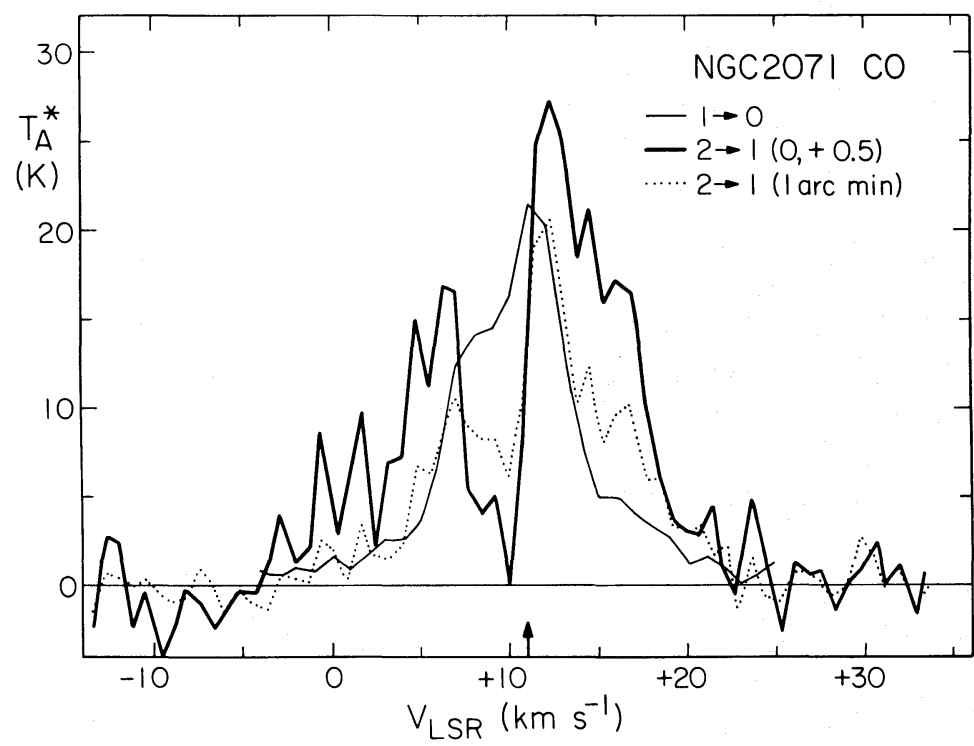

FIG. 8.-Profiles of the CO (1-0) and (2-1) lines observed toward NGC 2071. The CO (2-1) profiles shown are those observed at position $\delta+30^{\prime \prime}$, and the profile for the central position smoothed to a resolution of $1^{\prime}$. The velocity given by the ${ }^{13} \mathrm{CO}$ and $\mathrm{C} \alpha$ lines is indicated.

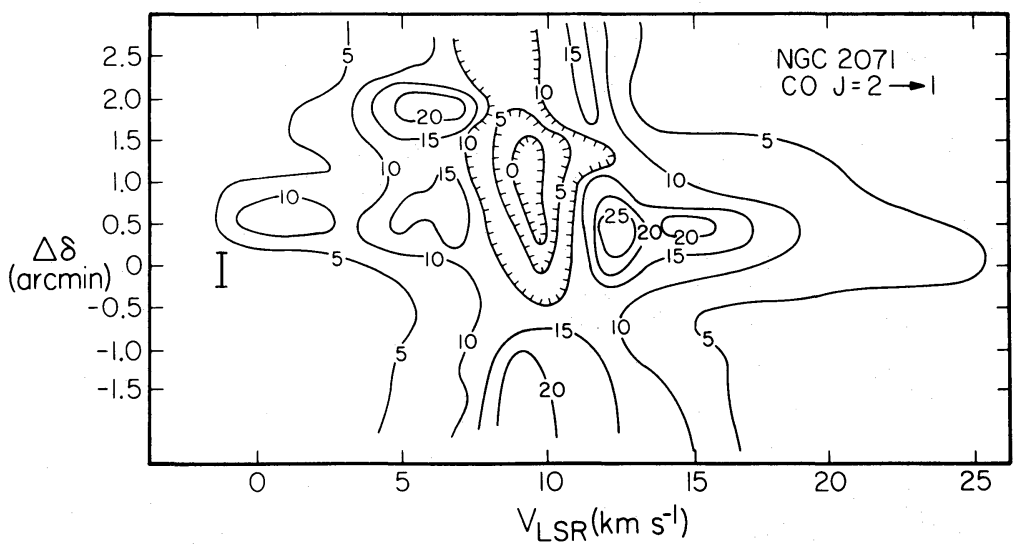

FIG. 9.- Velocity-declination map in the CO (2-1) line for NGC 2071

near the region where the velocity extent of the $\mathrm{CO}$ emission is largest (Fig. 9). It is likely that this feature and the $\mathrm{H}_{2} \mathrm{O}$ maser arise in a region of heated, expanding gas surrounding a newly formed early-type star. The velocity of the absorption dip is $9 \mathrm{~km} \mathrm{~s}^{-1}$, lower than the value of $11 \mathrm{~km} \mathrm{~s}^{-1}$ for the dense part of the cloud (Milman et al. 1975; Brown et al. 1975).

Mon R2 is also a molecular could containing an embedded B star. For this cloud, the velocity of the CO absorption dip is higher than that of the ${ }^{13} \mathrm{CO}$ line. This has been interpreted by Loren, Peters, and Vanden Bout (1974), Snell and Loren (1977), and Leung and Brown (1977) as due to the collapse of the front face of the molecular cloud towards the $\mathrm{H}$ iI region.
For both of these sources, the $\mathrm{CO}$ in the absorbing gas is subthermal, as shown by the greater depth of the CO (2-1) absorption dip relative to the (1-0) dip in both cases. These sources resemble DR 21 , except for the greater opacity of the absorbing $\mathrm{CO}$.

\section{c) $W 49 N$ and $W 51 A$}

The CO (2-1) and (1-0) line profiles for these sources are shown in Figures 13 and 16, and maps of the (2-1) distribution in Figures 14, 15, 17, and 18. For both of these sources, optically thick CO self-absorption is present; the cloud is fairly small for W49 and larger than the mapped area for W51. In both cases, the appearance 


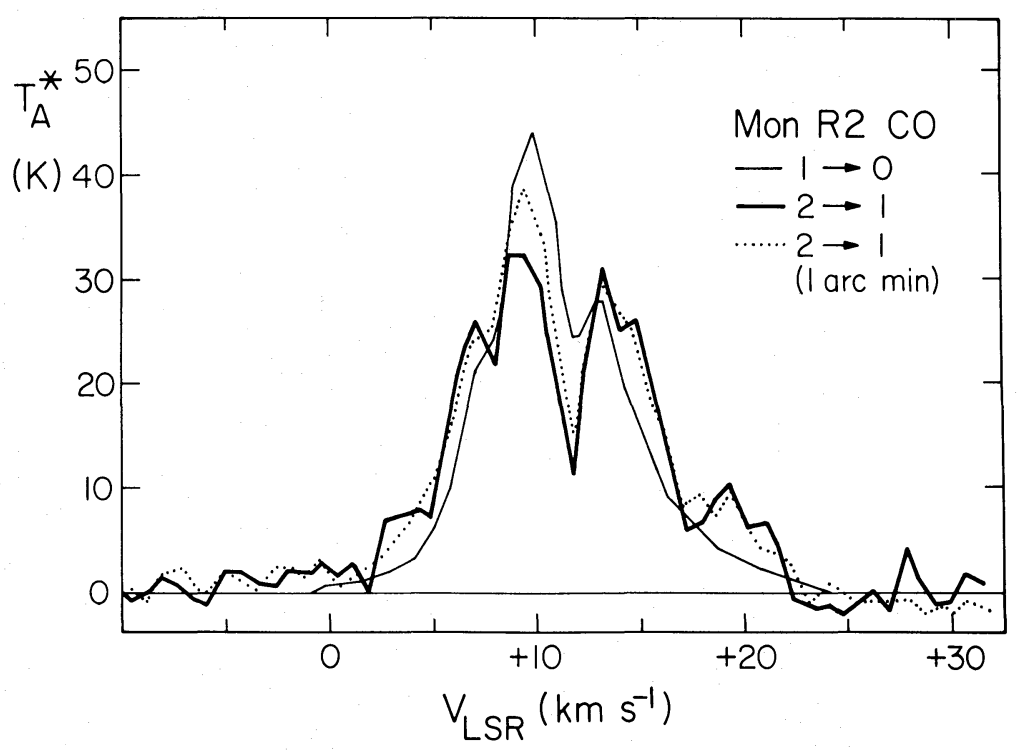

FIG. 10.- Profiles of the CO (1-0) and (2-1) lines observed toward Mon R2. The observed (2-1) profile, plus that smoothed to a resolution of $1^{\prime}$, are shown.

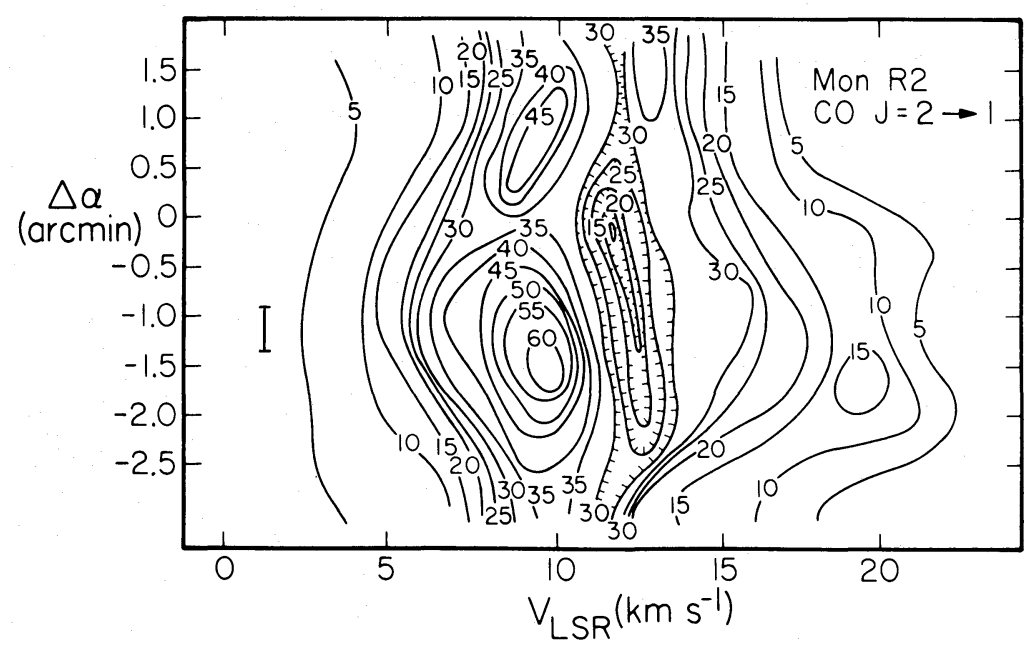

FIG. 11.-Velocity-right ascension map for the CO (2-1) emission from Mon R2

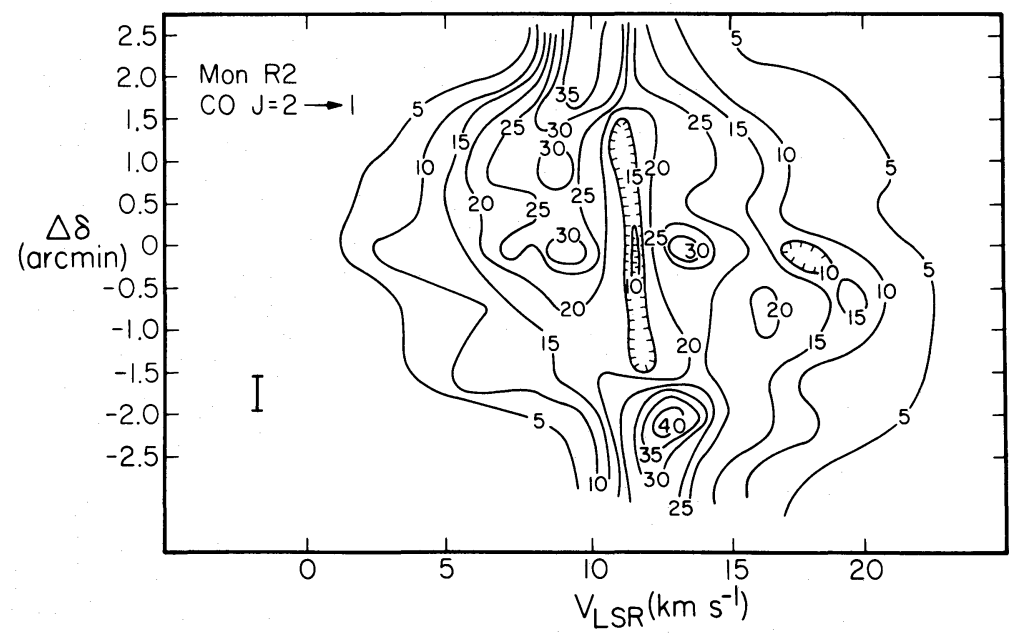

FIG. 12.-Velocity-declination map for the CO (2-1) emission from Mon R2 


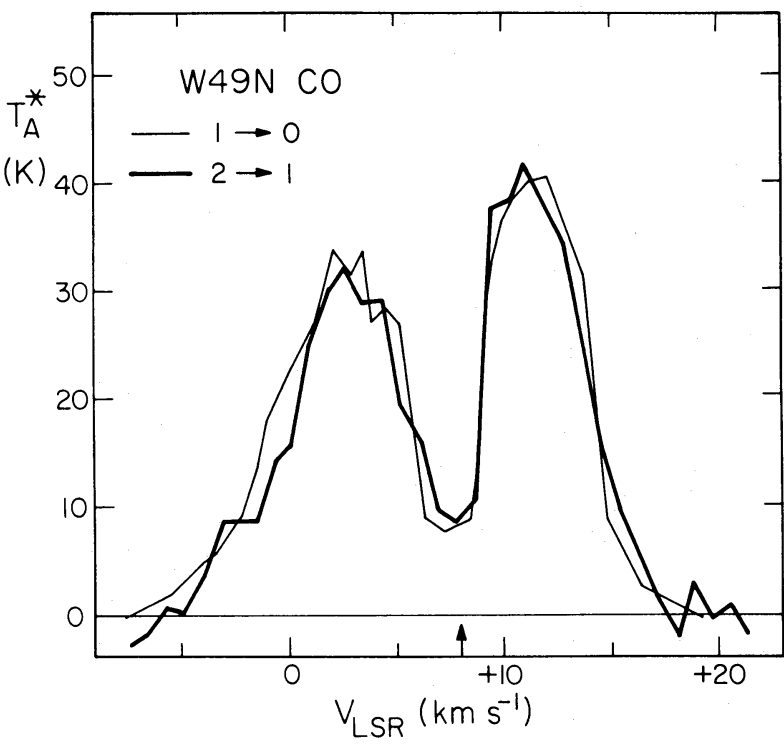

FIG. 13. - $\mathrm{CO}(1-0)$ and $(2-1)$ profiles observed toward W49N The velocity of the ${ }^{13} \mathrm{CO}(1-0)$ line is indicated.

of the maps suggests that the absorption is due to detached clouds lying in front of the sources.

For W49, Figure 13 shows that all components of the $\mathrm{CO}$ emission are optically thick at all velocities. The ${ }^{13} \mathrm{CO}(1-0)$ emission in this direction is present over the whole range of observed ${ }^{12} \mathrm{CO}$ emission and is centered at a velocity of $8 \mathrm{~km} \mathrm{~s}^{-1}$ (Mufson and Liszt 1977). These authors have also observed $2 \mathrm{~cm} \mathrm{H}_{2} \mathrm{CO}$ absorption against the source extending from 0 to $20 \mathrm{~km} \mathrm{~s}^{-1}$, and $\mathrm{H}$ recombination line emission at $+8 \mathrm{~km} \mathrm{~s}^{-1}$. Mufson and Liszt interpret the CO data as showing the presence of two emission components. However, since our data show that the ${ }^{12} \mathrm{CO}$ emission is optically thick, the ${ }^{13} \mathrm{CO}$ profile should have the same shape as the ${ }^{12} \mathrm{CO}$ profile. Since it does not, and peaks at $8 \mathrm{~km} \mathrm{~s}^{-1}$, the data suggest rather that the dip at $+8 \mathrm{~km} \mathrm{~s}^{-1}$ in the ${ }^{12} \mathrm{CO}$ profiles is due to optically thick absorption by a cold foreground cloud.

The case of W51 is somewhat different. There is a large $(10 \times 10 \mathrm{pc}$, Figs. 17 and 18$)$, cold $\left(T_{x} \sim 10 \mathrm{~K}\right)$, optically thick cloud in this direction producing absorption over the whole face of the molecular cloud at a velocity of $+66 \mathrm{~km} \mathrm{~s}^{-1}$. The $66 \mathrm{~km} \mathrm{~s}^{-1}$ component is prominent in the $\mathrm{H}_{2} \mathrm{CO}$ absorption against the source (Mufson and Liszt 1979) and the $\mathrm{H} \mathrm{I}$ emission in this direction (Burton 1970). This component has a velocity higher than allowed by circular galactic rotation and is attributed to gas streaming along the outside of the Sagittarius spiral arm (Burton and Shane 1970). The appearance of the absorption dip on the maps and its association with the spiral arm gas stream show that it is probably not contiguous with the W51 molecular cloud complex but lies somewhere along the line of sight, much closer to the Sun.

The CO (2-1) maps and profiles for W51 suggest the presence of a second, weaker self-absorption component at $V=+52 \mathrm{~km} \mathrm{~s}^{-1}$ (see especially Fig. 17), probably due to gas more closely associated with the W51 region. The ${ }^{13} \mathrm{CO}(1-0)$ line has a peak at this velocity (Mufson and Liszt 1979).

\section{d) $W 3$}

With the exception of DR 21, the molecular clouds described above probably have self-absorption produced

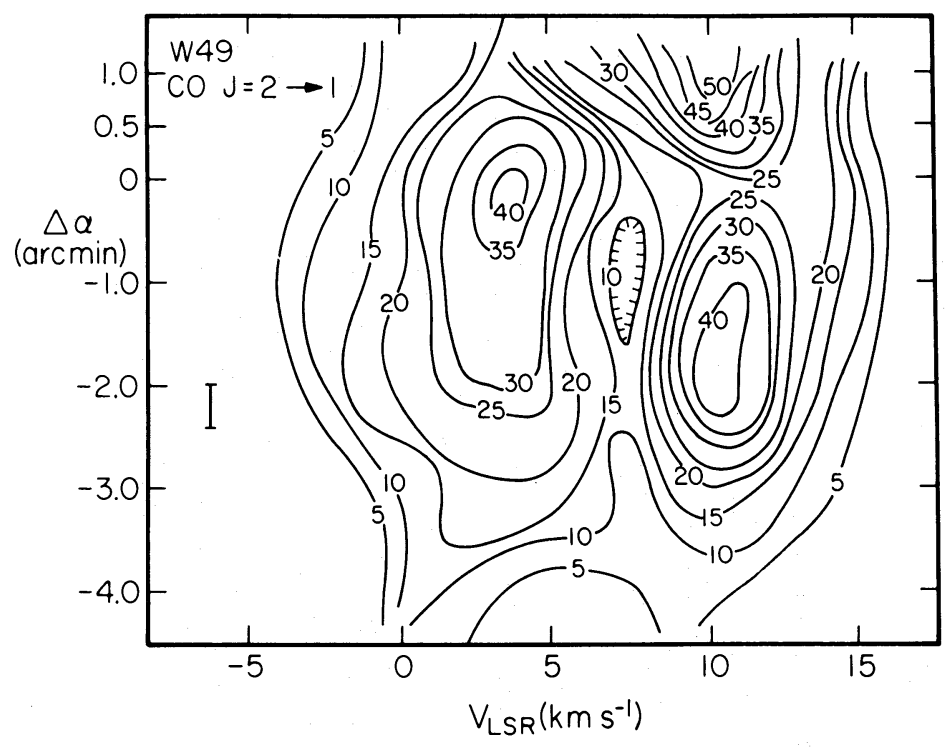

FIG. 14. - Velocity-right ascension map for the CO (2-1) emission from W49N 


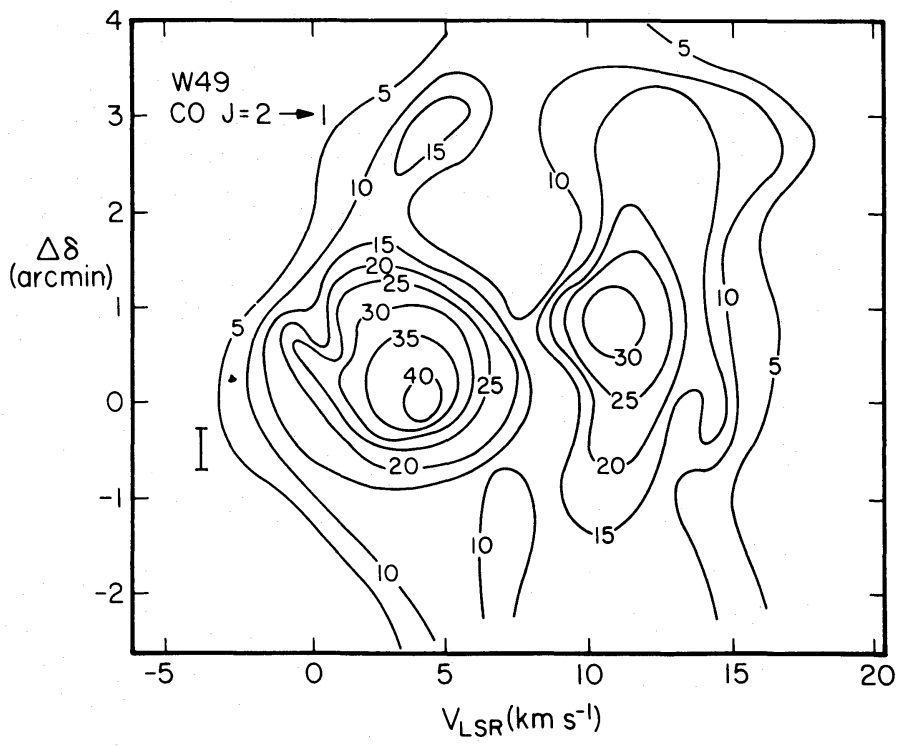

FIG. 15. - Velocity-declination map for the CO (2-1) emission from W49N

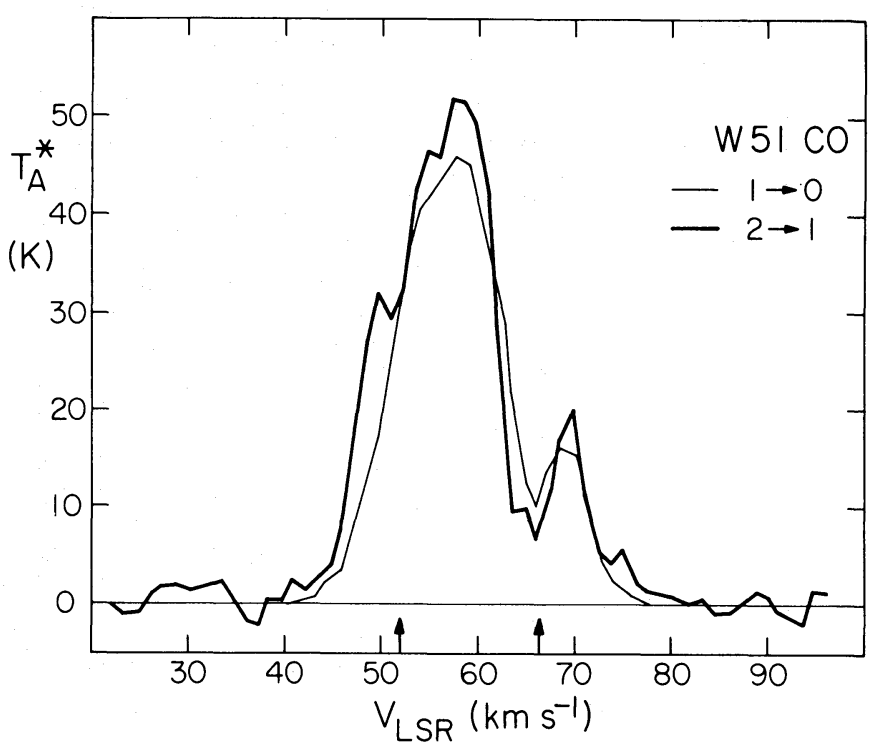

FIG. 16. - $\mathrm{CO}(1-0)$ and $(2-1)$ line profiles observed toward W51A. The velocities of the ${ }^{13} \mathrm{CO}$ line are indicated.

by the same situation: a cold, separate cloud in front of a hotter molecular cloud. In all cases except W51, the absorbing cloud is probably part of the same complex as the emitting region. The case of W3, however, appears to be different from all of the above clouds.

The profiles of the (1-0), $(2-1)$, and (3-2) lines are shown in Figure 19, and the maps of the CO (2-1) and (3-2) emission in Figures 20-23. These maps show that the $-38 \mathrm{~km} \mathrm{~s}^{-1}$ absorption dip is present over essentially the whole heated region. The dip has an apparent shift in velocity due, perhaps, to the changing temperature of the background; its mean velocity is close to that of the $\mathrm{CO}$ emission away from the heated region. The qualitative similarity of the maps to the model map of Figure $4 b$ suggests that the cold gas is physically associated with the W3 CO cloud, forming the colder outer regions of the hot cloud. This is the situation often invoked to explain self-absorption dips (cf. Snell and Loren 1977).

The precise relative depths of the reversals in the 


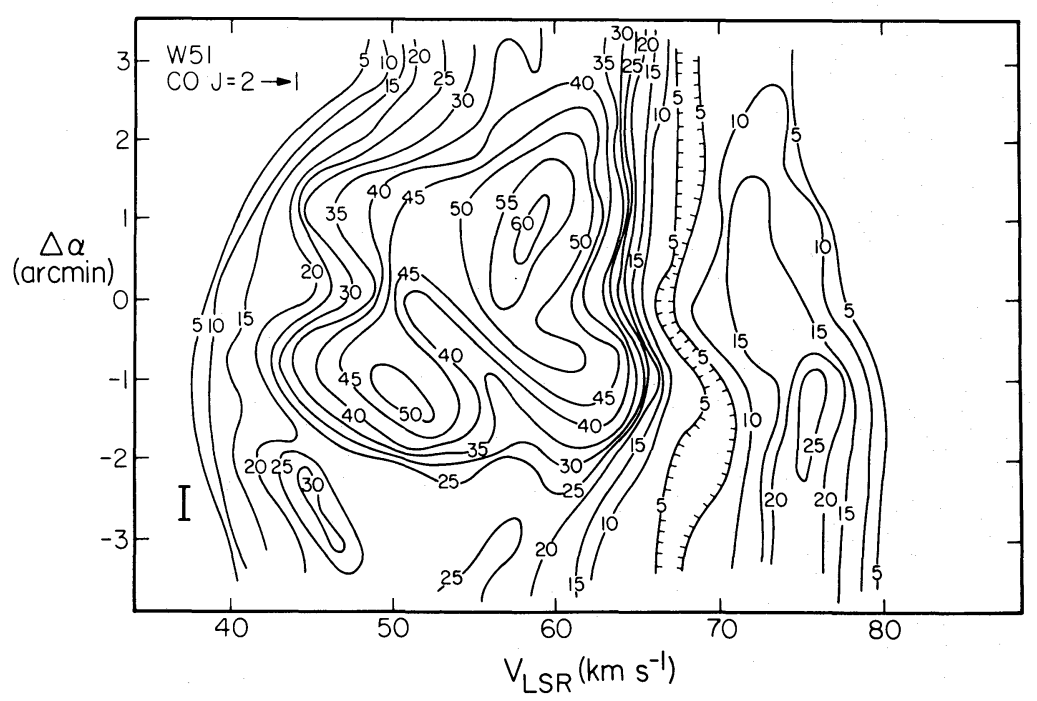

FIG. 17.- Velocity-right ascension map in the CO (2-1) line for W51A

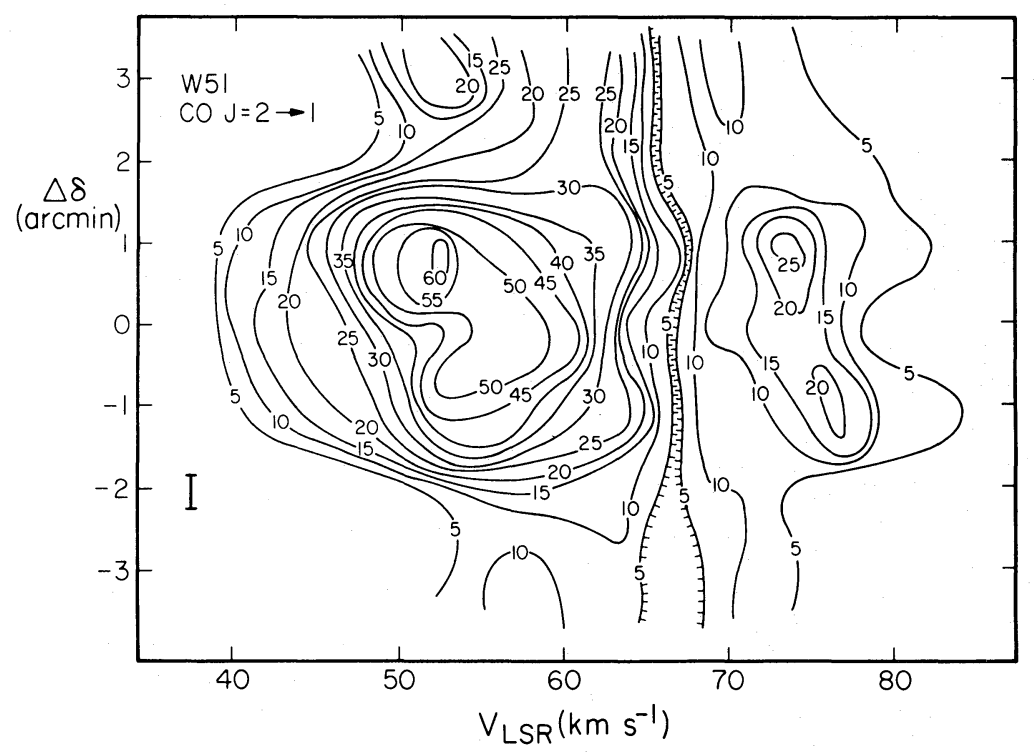

FIG. 18. - Velocity-declination map in the CO (2-1) line for W51

different $\mathrm{CO}$ lines (Fig. 19) cannot be reproduced by an LTE model. Use of a non-LTE model gives a reasonable fit with $T_{k}=35 \mathrm{~K}, T_{\mathrm{BG}}=65 \mathrm{~K}$, and $n\left(\mathrm{H}_{2}\right) \approx 10^{3}$ $\mathrm{cm}^{-3}$. The true brightness temperature of the gas associated with the $\mathrm{H}$ II region is thus much higher than would be found if the $\mathrm{CO}$ self-absorption dip were not taken into account.

The peak emission in the optically thin ${ }^{13} \mathrm{CO}(1-0)$ line occurs at a velocity of $-38 \mathrm{~km} \mathrm{~s}^{-1}$, while the dip in the $\mathrm{CO}(1-0)$ line is at $-36.5 \mathrm{~km} \mathrm{~s}^{-1}$. Snell and Loren
(1977) argued from this that the CO cloud is collapsing, a conclusion supported by the velocity of the $\mathrm{H}$ il region itself, $-43 \mathrm{~km} \mathrm{~s}^{-1}$ (Pedlar and Davies 1972). Our data for the $\mathrm{CO}(1-0),(2-1)$, and (3-2) lines show a progression with line opacity toward a velocity of $-38.5 \mathrm{~km}$ $\mathrm{s}^{-1}$. Comparison with Figure 2 shows that, if the cloud density is increasing along the line of sight toward the cloud, the cold region is expanding. The available data thus provide no clear-cut distinction between systematic collapse and expansion motions in this cloud. 


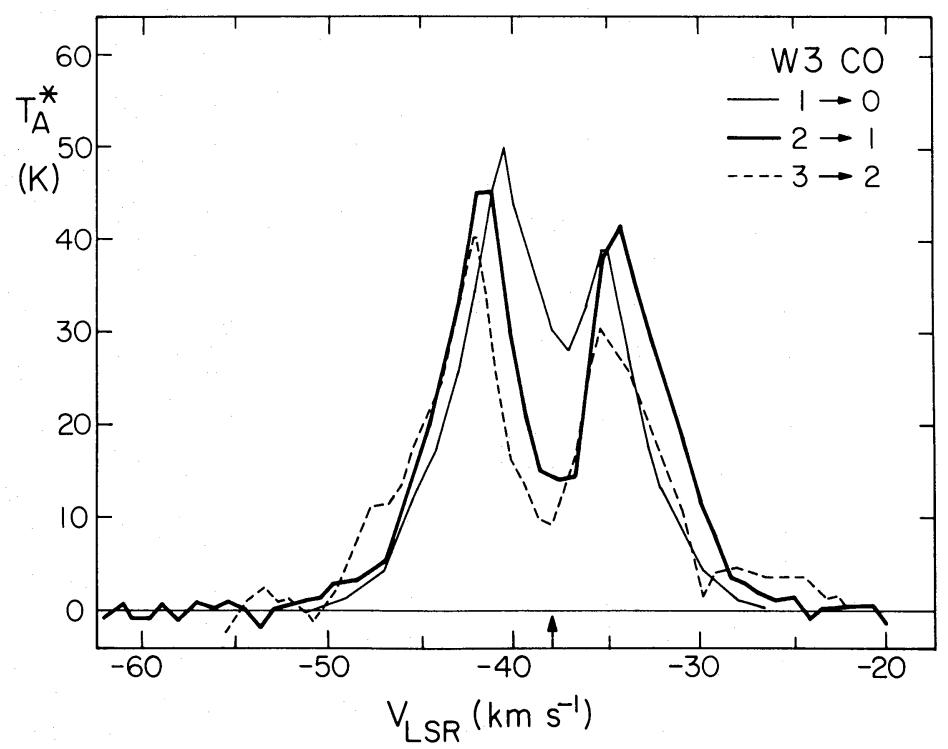

FIG. 19.- Profiles of the CO (1-0), (2-1), and (3-2) lines observed toward W3. The velocity of the ${ }^{13} \mathrm{CO}(1-0)$ line is shown.

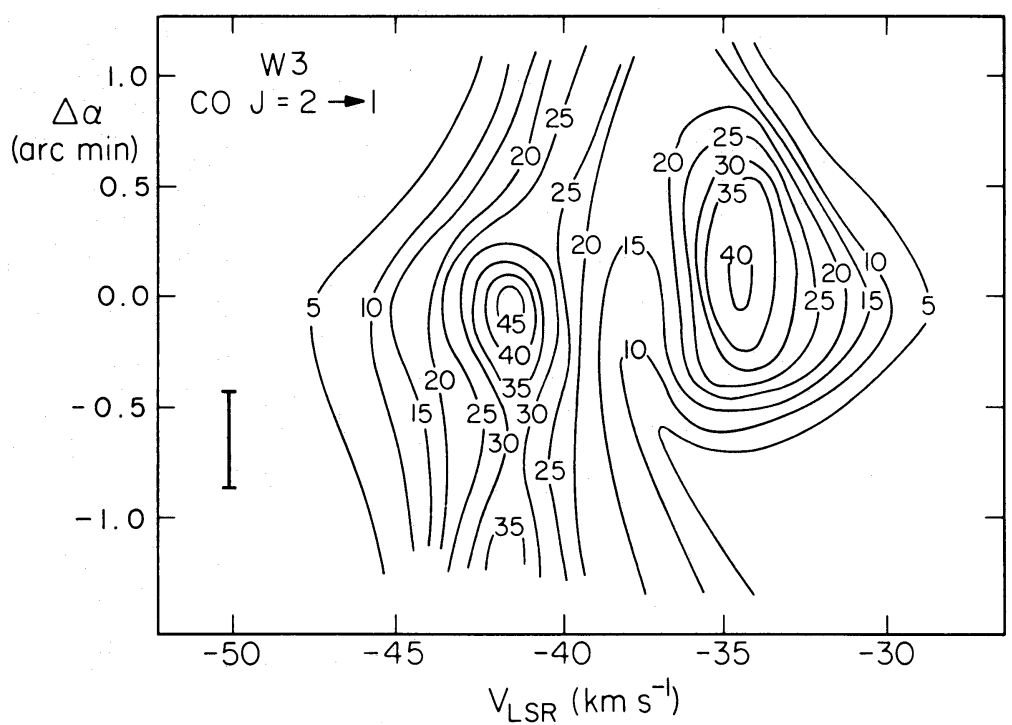

FIG. 20.-Velocity-right ascension map for the CO (2-1) line profiles observed toward W3

\section{e) NGC 1333, CRL961, $\rho$ Oph, and Cep A}

For NGC 1333, we observed only a single CO (2-1) profile, shown in Figure 24, where it is compared with the CO (1-0) profile of Loren (1976). The velocity of the dip in the $(2-1)$ line is close to that of the ${ }^{13} \mathrm{CO}$ (1-0) line. The absorption probably arises in the dark cloud covering the bright reflection nebulosity of NGC 1333.

The CO (2-1) profile for CRL 961, compared with the (1-0) profile of Blitz (1980), is shown in Figure 25. The absorbing cloud appears to be optically thick and to have a very low excitation temperature $(\sim 4 \mathrm{~K})$. The velocity of the dip $\left(+12.5 \mathrm{~km} \mathrm{~s}^{-1}\right)$ is lower than the $+14 \mathrm{~km} \mathrm{~s}^{-1}$ of the ${ }^{13} \mathrm{CO}$ emission. The discrepancy between the intensities of the CO (2-1) and (1-0) observations is too great to allow any conclusions to be drawn about this source.

The CO (2-1) profile for $\rho$ Oph is shown in Figure 26. The velocity of the bright, narrow emission spike $\left(+3 \mathrm{~km} \mathrm{~s}^{-1}\right)$ is close to that found for ${ }^{13} \mathrm{CO}$ and $\mathrm{H}_{2} \mathrm{CO}$ emission from the cloud (Loren, Evans, and Knapp 1979). In the observed direction, there is a large velocitydispersion, hotter region, which, since it is absorbed by 


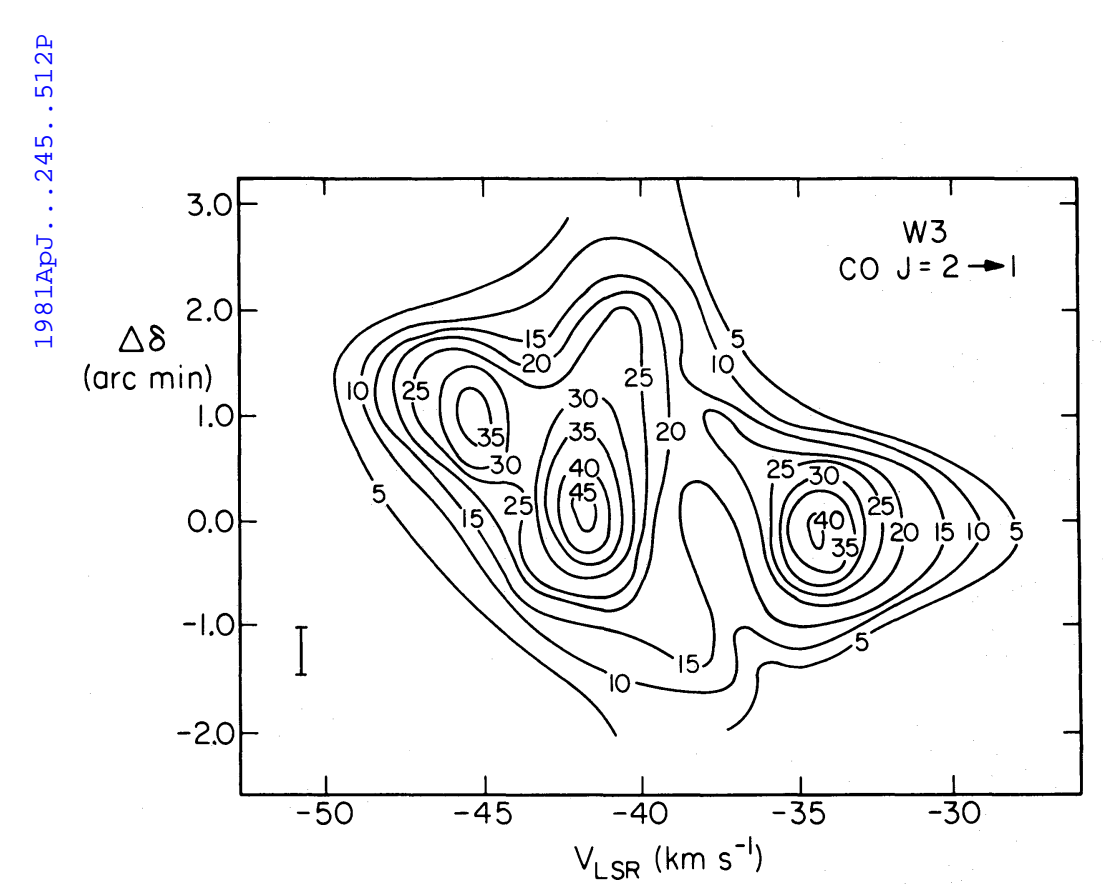

FIG. 21.- Velocity-declination map for the CO (2-1) observations of W3

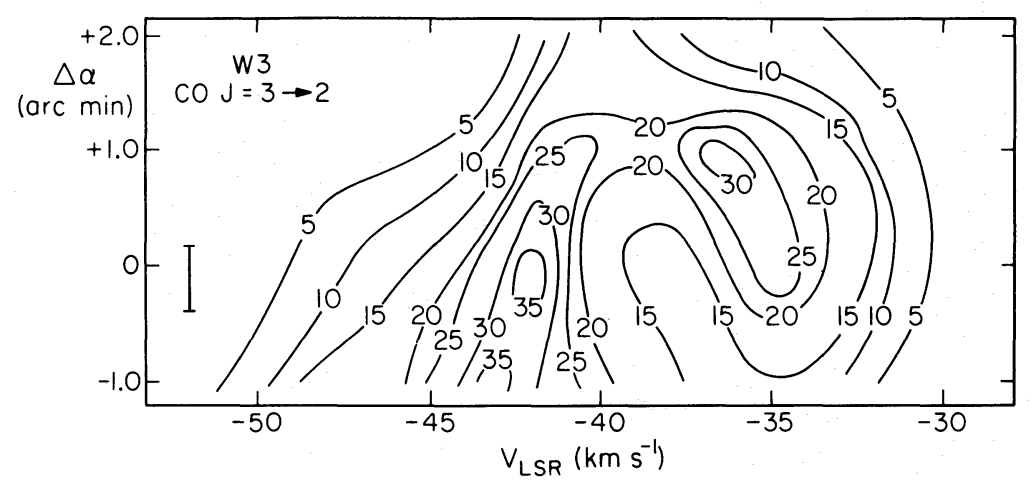

FIG. 22.-Velocity-right ascension map in the CO (3-2) line for W3

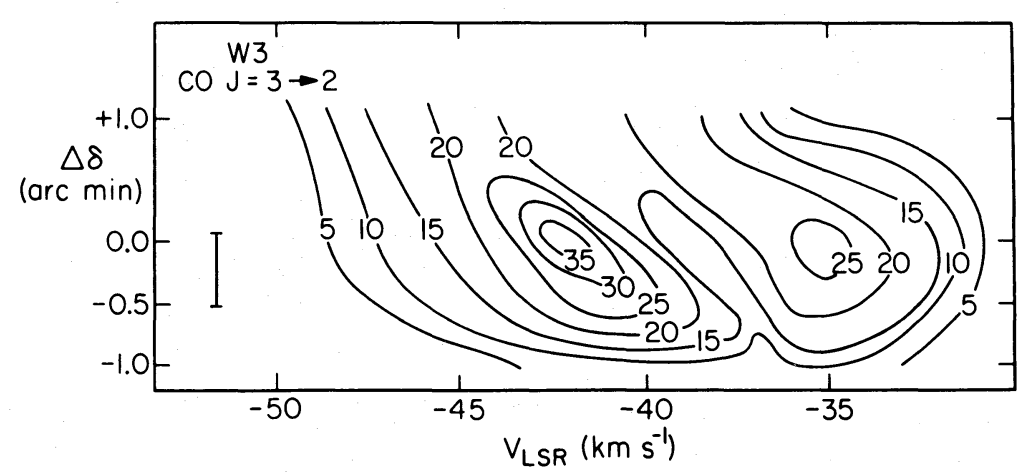

FIG. 23. - Velocity-declination map in the CO (3-2) line for W3 


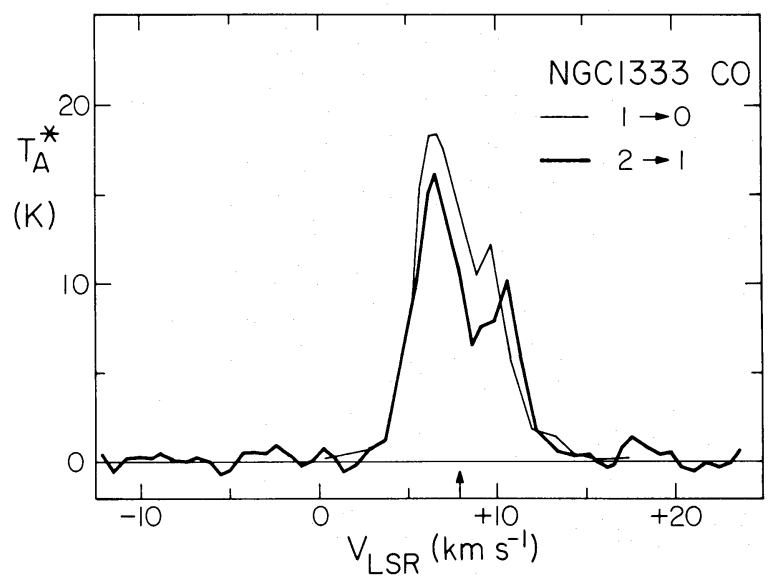

FIG. 24.- Profiles of the $\mathrm{CO}(1-0)$ and $(2-1)$ lines observed toward NGC 1333. The velocity of the ${ }^{13} \mathrm{CO}(1-0)$ line is indicated.

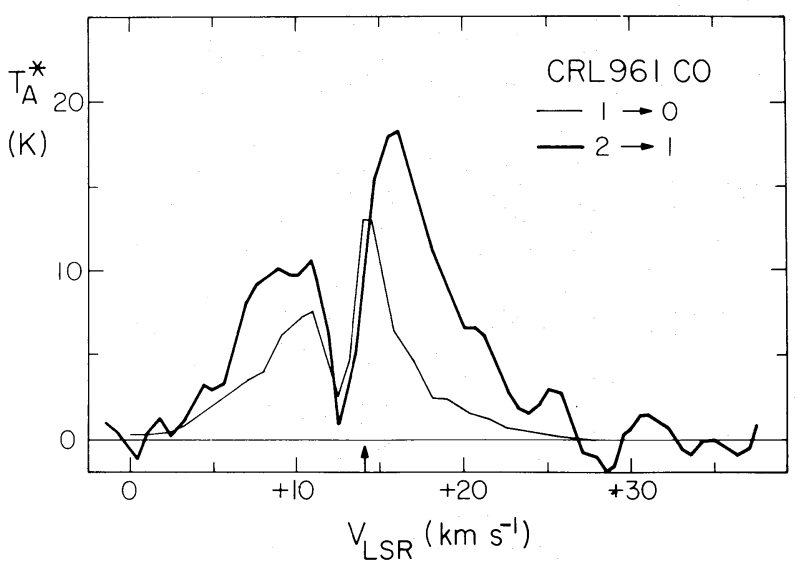

FIG. 25.-Profiles of the CO (1-0) and (2-1) lines observed toward CRL 961 . The velocity of the ${ }^{13} \mathrm{CO}(1-0)$ line is indicated.

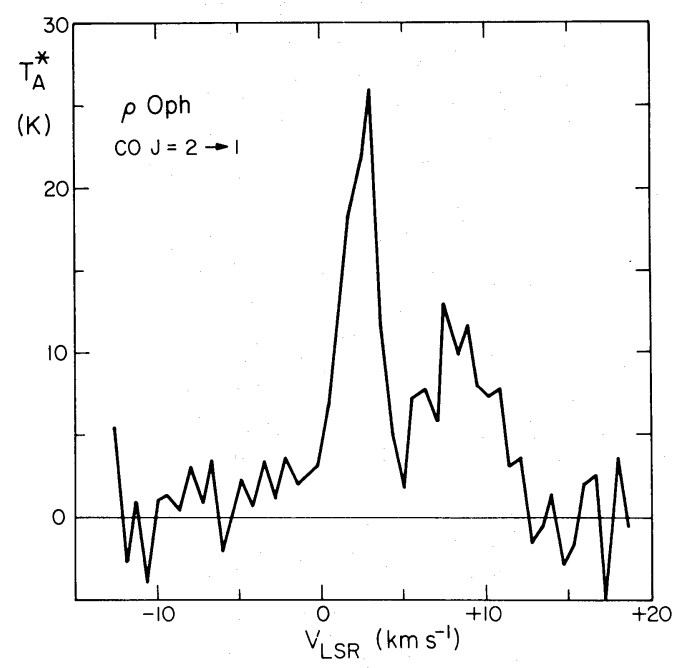

FIG. 26.- $\mathrm{CO}(2-1)$ line profile for the $\rho$ Oph cloud the high-velocity wing of the cold cloud component (cf. Phillips et al. 1977, Fig. 3b) is embedded in the larger cloud.

The observations of Cep A are shown in Figures 27-29. The appearance of the mapping observations suggests that the absorbing material is a separate, cold, low-density foreground cloud, similar to the case of NGC 2071 and Mon R2. The large discrepancy between the $(2-1)$ and $(1-0)$ data, however, makes detailed comparisons impossible. Away from the central, hightemperature region of the cloud (offsets of $3^{\prime}$ ), where the $\mathrm{CO}$ temperatures and line widths are those typical of a dark cloud, the absorption persists. This cloud provides the first observed example of self-absorption for lines from a cold dust cloud.

The observations described above show that $\mathrm{CO}$ selfabsorption is widespread, and that in all observed cases, the dip becomes deeper as the line frequency increases. The qualitative properties of the cold absorbing clouds are summarized in Table 2 . In most of the cases, the absorption appears to be due to a separate cold, lowdensity cloud in front of a hotter cloud. These clouds are probably usually associated with molecular cloud complex: for W51, however, absorption is produced by a distant cloud along the line of sight. For W3, in contrast to the above cases, the evidence suggests that the absorbing gas is the outside layer of the hotter molecular cloud.

In many cases, the $\mathrm{CO}$ in the absorbing gas is subthermally excited, suggesting that the total density and column density are low. For such clouds, the excitation temperature of the CO line is low $\left(T_{21} \lesssim 5 \mathrm{~K}\right.$ in some cases). In no case, however, do we find evidence for gas which is physically very cold (i.e., $T_{k} \lesssim 5 \mathrm{~K}$ ), although some of the CO self-absorption dips toward Sgr A (Liszt et al. 1977) suggest the presence of such gas in the Galaxy.

The comparison of our very simple qualitative models with the available data suggests that self-absorption in the $\mathrm{CO}$ lines is more common than has been recognized and therefore that the cloud temperatures measured by the $\mathrm{CO}$ lines may be underestimated. In the next section, an attempt is made to derive more accurate values for the core temperatures of molecular clouds using these models.

\section{THE CO TEMPERATURES FOR MOLECULAR CLOUDS}

The simple models described in § III and applied to the observations ( $(\mathrm{IV})$ show that the temperature of the background gas for many of the observed molecular clouds is considerably higher than would be inferred were self-absorption not taken into account. Since the background gas temperature is that of the gas immediately around the heating source, it is clear that the gas temperature in these star-forming regions has often 


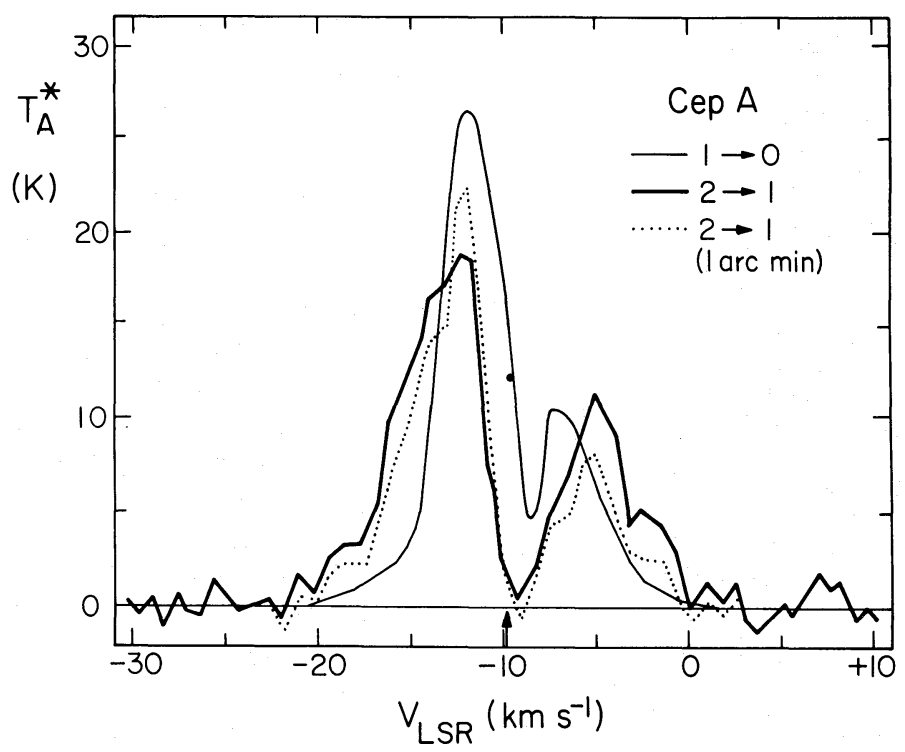

FIG. 27.- CO (1-0) and (2-1) line profiles observed toward Cep A. The velocity of the ${ }^{13} \mathrm{CO}(1-0)$ line is indicated.

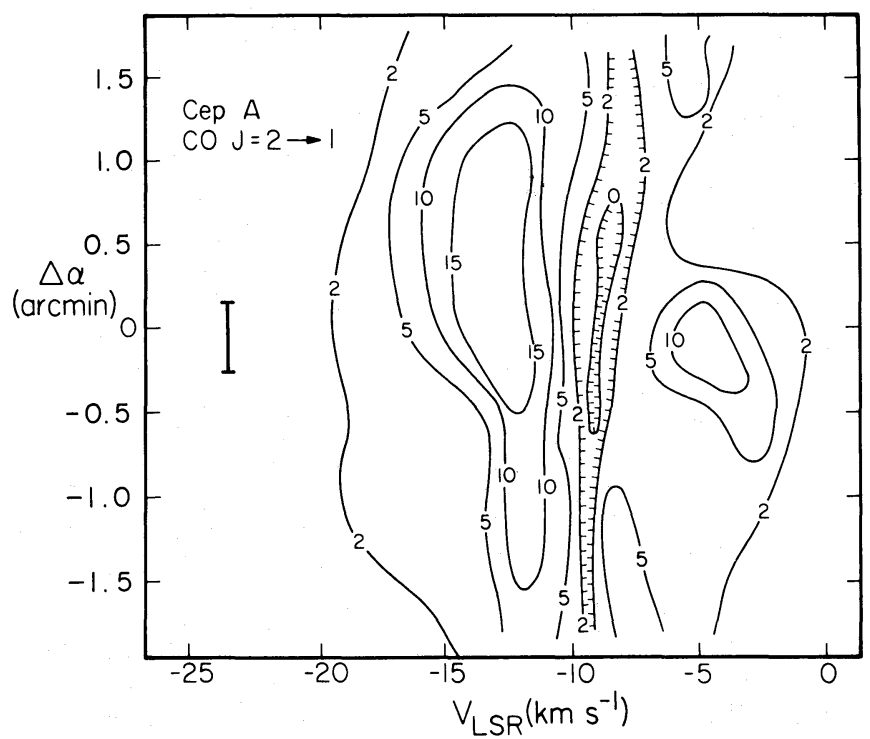

FIG. 28. - Velocity-right ascension map for the CO (2-1) emission from Cep A

TABLE 2

Properties of Cold Clouds

\begin{tabular}{|c|c|c|c|}
\hline Cloud & $\begin{array}{c}V(\operatorname{dip}) \\
\left(\mathrm{km} \mathrm{s}^{-1}\right)\end{array}$ & $\begin{array}{c}V \text { (hot cloud }) \\
\left(\mathrm{km} \mathrm{s}^{-1}\right)\end{array}$ & Absorbing Gas \\
\hline W3 & -38 & -38 & Slightly subthermal; $T_{K} \sim 35 \mathrm{~K}$; cloud exterior \\
\hline NGC $1333 \ldots$ & +9 & +8 & $T_{K} \sim 10 \mathrm{~K}$; separate clump \\
\hline NGC $2071 \ldots$ & +9 & +11 & Subthermal; separate clump \\
\hline Mon R2 ....... & +11.7 & +10.8 & Thermalized; separate clump \\
\hline CRL $961 \ldots$. & +12.5 & +14 & $T_{K} \sim 5 \mathrm{~K}$ \\
\hline$\rho \mathrm{Oph} \ldots \ldots \ldots$ & +6 & +3.5 & Subthermal; separate clump \\
\hline W $49 \mathrm{~N} \ldots \ldots \ldots$ & +7.5 & +8 & Thermalized; optically thick; $T_{K} \sim 13 \mathrm{~K}$; separate cloud \\
\hline W51A ........ & +66 & +60 & Thermal; optically thick; separate clump \\
\hline DR $21 \ldots \ldots \ldots$ & -3.0 & -3.5 & Subthermal; separate clump (?) \\
\hline Cep A $\ldots \ldots \ldots$ & -9.2 & -10 & Subthermal; $T_{21}$ low; separate clump \\
\hline
\end{tabular}




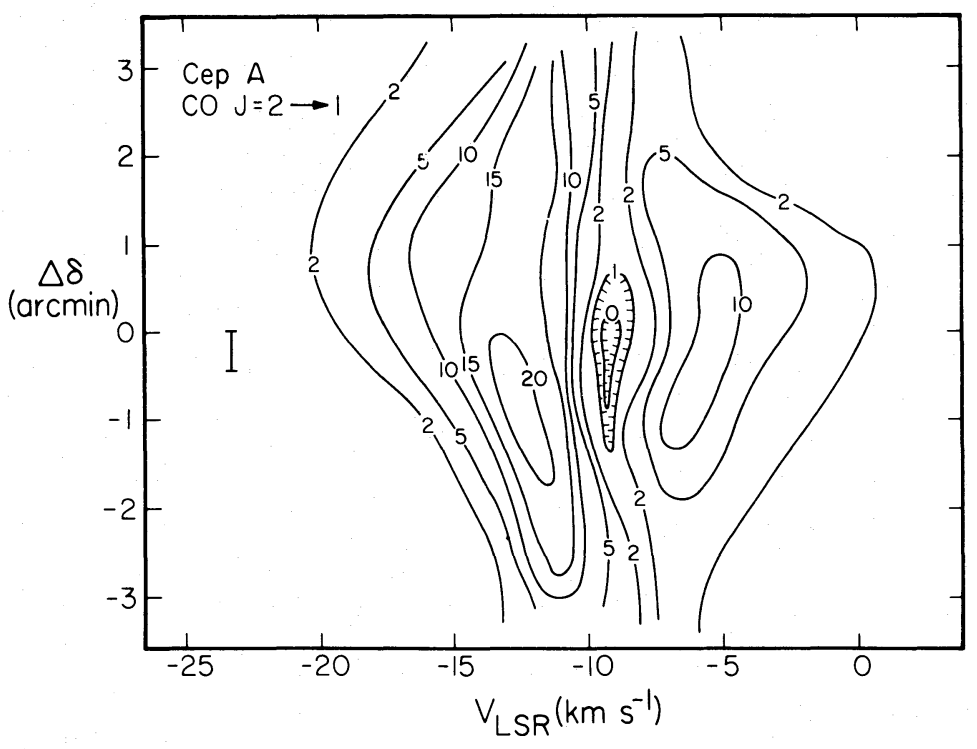

FIG. 29.-Velocity-declination map for the CO (2-1) emission from Cep A

been severely underestimated. Also, if the peak temperature of a line has been underestimated, the line width has been overestimated. In Table 3, we list the brightness temperatures of the background clouds, corrected for absorption using our observations. We also list corrected values of the half-power line width $\Delta V$ for the $\mathrm{CO}$ (1-0) line for each source. Excluded from Table 3 are CRL 961 and $\rho$ Oph, for which our molecular line information is inadequate. Also listed in Table 3 is the dust temperature for each source, where available, found from far-infrared data. The dust temperatures in Tables 3 are, with the exception of $\mathrm{W} 49 \mathrm{~N}$, calculated from the ratio of fluxes around $50 \mu$ and $100 \mu$, assuming a $1 / \lambda$ emissivity law for the grains. The quoted uncertainties in the grain temperatures are based on a typical absolute uncertainty of $20 \%$ in the measured flux ratio. The uncertainties in the values of $T_{B}(\mathrm{CO})$ are found from the values of $T_{B}$ estimated for the individual $\mathrm{CO}$ lines. Within the errors, the gas and dust temperatures agree, although the gas temperatures are on the whole some- what lower than the dust temperatures. The agreement is much better than would be found were the $\mathrm{CO}$ temperatures not corrected for the effects of selfabsorption. The agreement between the $\mathrm{CO}$ and the grain temperatures shows that, averaged over regions of $\sim 30^{\prime \prime}-1^{\prime}$, the gas and dust are close to thermal equilibrium in many sources.

The apparently lower gas temperatures found when self-absorption effects in the $\mathrm{CO}$ lines are ignored have been attributed by several authors (e.g., Goldreich and Kwan 1974; Scoville and Kwan 1976) to low gas densities or to inefficient gas-grain coupling in the vicinity of the heating source. However, our data show that the difference between the measured gas and dust temperatures can be largely resolved by properly taking into account the effects of self-absorption in the $\mathrm{CO}$ lines and suggest that the gas temperatures derived from the brightness temperatures of $\mathrm{CO}$ lines be treated with caution, unless transitions with several $J$-values are available.

TABLE 3

Brightness Temperatures and CO $(1-0)$ Line Widths for Molecular Clouds

\begin{tabular}{|c|c|c|c|c|}
\hline Cloud & $\begin{array}{c}T_{B} \\
(\mathrm{~K})\end{array}$ & $\begin{array}{l}\Delta V(1-0) \\
\left(\mathrm{km} \mathrm{s}^{-1}\right)\end{array}$ & $\begin{array}{l}T \text { (dust) } \\
\quad(\mathrm{K})\end{array}$ & Reference \\
\hline W3 & $66 \pm 5$ & 8 & $65 \pm 7$ & Werner et al. 1981 \\
\hline NGC $1333 \ldots$ & $24 \pm 7$ & 5 & $\ldots$ & \\
\hline NGC $2071 \ldots$ & $34 \pm 8$ & 6 & $40 \pm 3$ & Harvey et al. 1979 \\
\hline Mon R2 ...... & $50 \pm 8$ & 7 & $55 \pm 5$ & Thronson et al. 1980 \\
\hline W49N & $51 \pm 5$ & 14 & $52 \pm 4$ & Erickson and Tokunaga 1980 \\
\hline W51A. & $50 \pm 6$ & 19 & $50 \pm 4$ & Thronson and Harper 1979 \\
\hline DR 21 & $44 \pm 7$ & 7 & $47 \pm 4$ & Harvey et al. 1977 \\
\hline Cep A ........ & $33 \pm 11$ & 7 & $41 \pm 3$ & Evans et al. 1981 \\
\hline
\end{tabular}




\section{CONCLUSIONS}

In this paper, we have described observations of the CO (2-1) and, in two cases, the (3-2) lines toward the cores of several star-forming molecular clouds. In several cases, the distribution of molecular emission has been mapped. The observations show:

1) Self-absorption in the $\mathrm{CO}$ lines appears to be more common than has previously been thought. In several cases, the absorption appears to be due to low-density foreground gas and becomes deeper in the higher frequency CO lines. Most striking is the case of DR 21, for which a clear self-reversed appearance is present only in the (3-2) line, showing that the (2-1) and (1-0) lines must also, despite their appearance, be strongly absorbed.

2) Because of absorption, the gas temperatures in the core regions of molecular clouds as measured using the $\mathrm{CO}$ lines may often be greatly underestimated. When corrections for absorption are applied, the average gas temperatures come into reasonable agreement with those of the dust.

3) Self-absorption appears to arise from a variety of geometric configurations, including a cold envelope around a hot core (W3) or a cold cloud well in front of, and detached from, the hotter background gas (W51).

4) The available evidence on the kinematics of large molecular clouds is conflicting as regards systematic collapse or expansion motions.

5) Several molecular clouds show structure on the size scale $\left(\sim 30^{\prime \prime}\right)$ of these observations.

We thank the staff of the Owens Valley Radio Observatory and of the Palomar Observatory for their help in support of this work. Research in millimeter-wave astronomy at the California Institute of Technology is supported by the National Science Foundation via grant AST-79-16815. Part of this work was supported by NASA grant NGL 05-002-207.

\section{REFERENCES}

Blitz, L. 1980, preprint.

Brown, R. L., Knapp, G. R., Kuiper, T. B. H., and Kuiper,

E. N. R. 1975, Ap. J. (Letters), 195, L23.

Burton, W. B. 1970, Astr. Ap. Suppl., 2, 291.

Burton, W. B., and Shane, W. W. 1970, in IAU Symposium 38, The Spiral Structure of Our Galaxy, ed. W. Becker and G. Contopoulos (Dordrecht: Reidel), p. 39.

Dickel, J. R., Dickel, H. R., and Wilson, W. J. 1978, Ap. J., 223, 840.

Erickson, E. F., and Tokunaga, A. T. 1980, Ap. J., 238, 596

Evans, N. J., et al. 1981, Ap. J., submitted.

Goldreich, P., and Kwan, J. 1974, Ap. J., 189, 441.

Harvey, P. M., Campbell, M. F., and Hoffman, W. F. 1977, Ap. J., 211, 786.

Harvey, P. M., Campbell, M. F., Hoffman, W. F., Thronson, H. A., and Gatley, I. 1979, Ap. J., 229, 990.

Israel, F. P., de Graauw, T., Lidholm, S., and Sargent, A. I. 1981, in preparation.

Kwan, J. 1978, Ap. J., 223, 147.

Leighton, R. B. 1978, Final Tech. Rept., NSF project AST73-04908.

Leung, C. M., and Brown, R. L. 1977, Ap. J. (Letters), 214, L73.

Leung, C. M., and Liszt, H. S. 1976, Ap. J., 208, 732.

Liszt, H. S., Burton, W. B., Sanders, R. H., and Scoville, N. Z 1977, Ap. J., 213, 38.

Liszt, H. S., Wilson, R. W., Penzias, A. A., Jefferts, K. B., Wannier, P. G., and Solomon, P. M. 1974, Ap. J., 190, 557.

Loren, R. B. 1976, Ap. J., 209, 466.

Loren, R. B., Evans, N. J., and Knapp, G. R. 1979, Ap. J., 234, 932 .
Loren, R. B., Peters, W. L., and Vanden Bout, P. A. 1974, Ap. J. (Letters), 194, L103.

Loren, R. B., and Wootten, H. A. 1980, Ap. J., 242, 568.

Milman, A. S., Knapp, G. R., Kerr, F. J., Knapp, S. L., and Wilson, W. J. 1975, A. J. 80, 93.

Mufson, S. L., and Liszt, H. S. 1977, Ap. J., 212, 664. 1979, Ap. J., 232, 451.

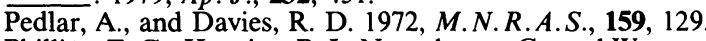

Phillips, T. G., Huggins, P. J., Neugebauer, G., and Werner, M. W. 1977, Ap. J. (Letters), 217, L161.

Phillips, T. G., Huggins, P. J., Wannier, P. G., and Scoville, N. Z. 1979, Ap. J., 231, 720 .

Sargent, A. I. 1977, Ap. J., 218, 736.

Schwartz, P. R., and Buhl, D. 1973, Ap. J. (Letters), 201, L27.

Scoville, N. Z., and Kwan, J. 1976, Ap. J., 206, 718.

Scoville, N. Z., and Solomon, P. M. 1974, Ap. J. (Letters), 187 L67.

Snell, R. L., and Loren, R. B. 1977, Ap. J., 211, 122.

Thronson, H. A., Gatley, I., Harvey, P. M., Sellgren, K., and Werner, M. W. 1980, Ap. J., 237, 66.

Thronson, H. A., and Harper, D. A. 1979, Ap. J., 230, 133

Ulich, B. L., and Haas, R. W. 1976, Ap. J. (Suppl.), 30, 247.

Werner, M. W., Gatley, I., Harper, D. A., Becklin, E. E., Lowenstein, R. F., Telesco, C. M., and Thronson, H. A. 1976, Ap. J., 204, 420.

Werner, M. W., et al. 1981, in preparation.

D. EnNis and G. Neugebauer: 320-47, California Institute of Technology, Pasadena, CA 91125

P. J. Huggins: Department of Physics, New York University, 4 Washington Place, New York, NY 10003

G. R. KNAPP: Department of Astrophysical Sciences, Princeton University, Princeton, NJ 08544

T. G. Phillips: 405-47, California Institute of Technology, Pasadena, CA 91125

P. G. WANnIER: 102-24, California Institute of Technology, Pasadena, CA 91125

M. W. Werner: NASA Ames Research Center, Moffett Field, CA 94035 